\title{
Role of first-neighbor geometry in the electronic and mechanical properties of atomic contacts
}

\author{
C. Sabater, ${ }^{1, *}$ W. Dednam,,${ }^{1,2}$ M. R. Calvo,,${ }^{1,3,4}$ M. A. Fernández, ${ }^{1}$ C. Untiedt, ${ }^{1}$ and M. J. Caturla ${ }^{1, \dagger}$ \\ ${ }^{1}$ Departamento de Física Aplicada, Universidad de Alicante, Campus de San Vicente del Raspeig, E-03690 Alicante, Spain \\ ${ }^{2}$ Department of Physics, University of South Africa, Science Campus, Florida Park, Johannesburg 1710, South Africa \\ ${ }^{3}$ CIC NanoGune, E-20018, Donostia, San Sebastian, Spain \\ ${ }^{4}$ Ikerbasque, Basque Foundation for Science, 48013 Bilbao, Spain
}

(Received 26 September 2017; revised manuscript received 30 January 2018; published 16 February 2018)

\begin{abstract}
We study in detail, via experimental measurements, atomistic simulations, and density functional theory transport calculations, the process of formation and the resulting electronic properties of atomic-sized contacts made of $\mathrm{Au}, \mathrm{Ag}$, and $\mathrm{Cu}$. Our data analysis of both experimental results and simulations leads to a precise relationship between geometry and electronic transmission-we reestablish the significant influence of the number of first neighbors on the electronic properties of atomic-sized contacts. This result allows us also to interpret subtle differences between the metals during the process of contact formation as well as the characteristics of the resulting contacts.
\end{abstract}

DOI: 10.1103/PhysRevB.97.075418

\section{INTRODUCTION}

Single atoms and molecules have been widely hailed as potential electronic devices over the past 20 years [1]. To make such devices a reality, metallic contact formation and the electrical characteristics of few-atom contacts need to be understood in depth at the atomic level. Electrical conduction in single-atom contacts has been broadly studied both from an experimental and a theoretical point of view [2], and single-atom contacts have been proposed as elementary circuit components, such as quantized resistors, capacitors [3], or switches [4].

The conductance of few-atom contacts is given by the sum of contributions from quantized transport modes propagating at the contact junction, and the number and transmission probabilities of those modes are, in turn, determined by the size and chemical valence of the central part of the constriction [2]. For example, both a single-atom contact and a monatomic chain of $\mathrm{Au}$ exhibit a resistance of around a quantum of conductance, $G_{0}=2 e^{2} / h$, which in this case is the signature of electronic transport through a single, fully open, quantum channel [5]. However, variations in the geometrical configuration of the leads [6], i.e., the number of neighboring atoms in the constriction, give rise to fluctuations of up to $20 \%$ in the conductance of a single atomic contact. Not only are the electrical properties of single atom contacts strongly influenced by their coordination to the leads, but their mechanical properties are as well. When two electrodes in the tunneling regime eventually come into contact, it is known, for certain materials and geometries, that the process of contact formation happens as a sudden jump. Nonetheless, jump to contact is not

*Corresponding author: carlos.sabater@ua.es; Present address: Chemical Physics Department, Weizmann Institute of Science, 76100 Rehovot, Israel.

†Corresponding author: mj.caturla@ua.es a generalized phenomenon, and the process of formation may be smooth [7]. The probability of the occurrence of a jump to contact and the details of this process have already been suggested to depend strongly not only on the bulk mechanical properties of the material, such as its cohesive energy and Young's modulus [8,9], but also, for certain materials, e.g., $\mathrm{Au}$ or $\mathrm{Cu}$, on the specific geometry of the contacting leads $[10,11]$.

In this article, we focus on the influence of the first-neighbor configurations on the process of formation of single-atom contacts made of $\mathrm{Au}, \mathrm{Ag}$, and $\mathrm{Cu}$, as well as their associated conductance values. Toward that end, we combine atomistic simulations and quantum transport calculations [12-17] with a detailed analysis of experimental results. We improve the statistical analysis carried out by Untiedt et al. [7] for $\mathrm{Au}$, and we compare our results with those obtained from the atomistic simulations we perform to determine the most likely first-neighbor structures at first contact, and the corresponding conductance values we calculate from density functional theory (DFT) methods [18-20]. From such a comparison between simulation and experimental results, we can relate the distribution of contact conductances to specific geometries. In agreement with the results published in Refs. [6,7], we find the most likely geometries to lie within four classes: monomers, dimers, double contacts (DCs), and triple contacts (TCs). Furthermore, we identify more specific structures within these classes, and more interestingly we find the dispersion in conductance values for each of these classes to be a consequence of the variations in the number of first neighbors. Our analysis provides a precise assignment of the conductance values reported for these configurations, and remarkably it yields a broader distribution of conductance values for the monomer than in previous works on $\mathrm{Au}$, ultimately explaining previous disagreements between experiments and theory. The reason for this can be traced to a higher dependence of the monomer's conductance on the number of first neighbors. We complete our study by carrying out a similar analysis for $\mathrm{Ag}$ and $\mathrm{Cu}$. 
(a)

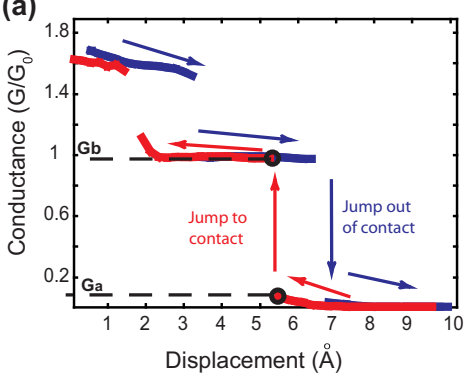

(b)

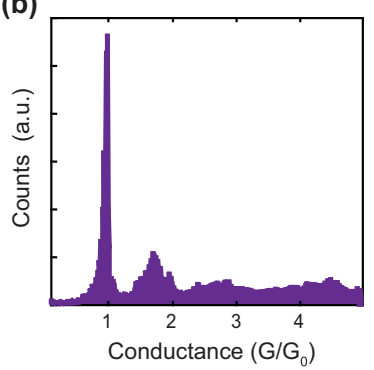

FIG. 1. (a) Conductance trace for the formation and rupture of a gold atomic contact recorded in our STM-MCBJ setup at $4.2 \mathrm{~K}$. (b) Conductance histogram built from more than $1000 \mathrm{Au}$ contact rupture traces.

\section{METHODS}

\section{A. Experimental methods}

Our atomic contacts are fabricated by performing several cycles of indentation and separation of two electrodes made of the same high-purity (99.999\%) metal- $\mathrm{Au}, \mathrm{Ag}$, or $\mathrm{Cu}$ - under cryogenic vacuum at $4.2 \mathrm{~K}$. The electrical conductance of the junctions (obtained as the measured current divided by the applied voltage of $100 \mathrm{mV}$ ) is recorded while the two electrodes are carefully brought into contact in a scanning tunneling microscope (STM) setup, as described in previous works [6,7]. The traces of conductance as a function of electrode distance [Fig. 1(a)] contain valuable information about the process of contact rupture and formation. When electrodes are close enough but not yet in contact, electrons may tunnel between them. In the tunneling regime, the conductance increases exponentially as the separation between leads decreases. The conductance increases smoothly until a sudden jump occurs, from the tunneling regime up to a clear plateau at around $1 G_{0}$, indicating the formation of a monatomic contact [2]. Examples of rupture and formation traces are displayed in Fig. 1(a).

Every realization of an atomic-size contact produces a slightly different conductance trace, which is suggestive of a variation in structural configurations. Therefore, a statistical analysis of the data is key to extracting information about the most probable configurations. An approach that is widely used in the literature [2] is the construction of a conductance histogram [such as the one in Fig. 1(b) for the case of rupture traces of $\mathrm{Au}$ ] to determine the conductance values associated with the most probable configurations of the single-atom contact.

A more specific method for the study of contact formation was introduced by Untiedt et al. [7]. As sketched in Fig. 1(a), for each formation trace, the highest jump in conductance between two consecutive points is monitored. Two conductance values are then recorded: $G_{a}$, from which the jump occurs, and $G_{b}$, the final value immediately after the jump. A density plot of the pairs $\left(G_{a}, G_{b}\right)$ (main panel in Fig. 2) displays the values of greatest probability from and to which the conductance jump occurs.

As mentioned above, prior to contact formation, the tunneling conductance depends exponentially on the distance between electrodes as $G \simeq K e^{-\frac{\sqrt{2 m \phi}}{h} d}$, where $K$ is a propor-

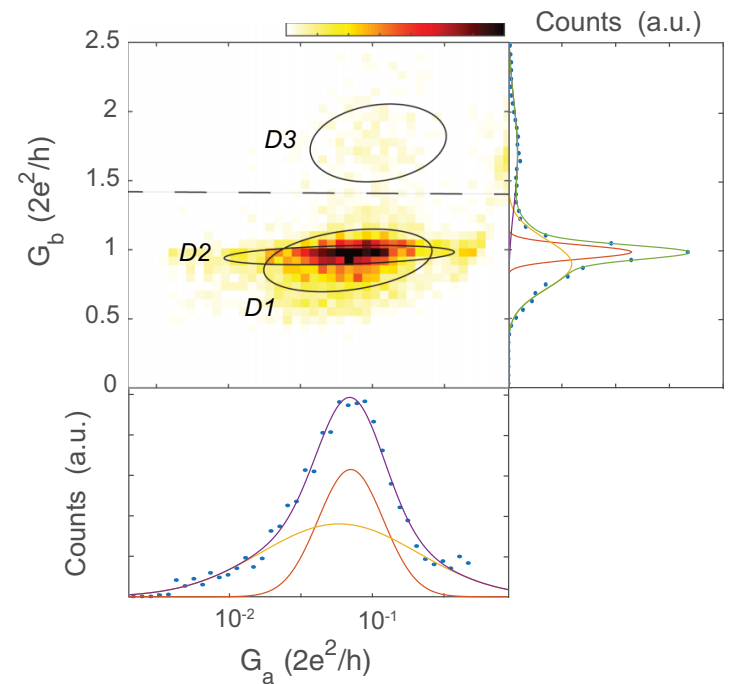

FIG. 2. Central panel: density plot constructed from the pairs $\left(G_{a}, G_{b}\right)$ obtained as described in the text from more than 2000 traces of formation of $\mathrm{Au}$ contacts. Right panel: Scatter plot showing the projection of the density plot on the $G_{b}$ axis. As shown in previous works [7], this projection can be fitted to the sum of three Gaussian peaks (green line). The purple, orange, and yellow lines represent the individual Gaussian components. Bottom panel: Projection of the density plot on the $G_{a}$ axis (scatter plot). The maximum above the dashed line has been left out of the analysis here in order to more clearly identify the components of the maximum below the line. The projection of the latter maximum can be fitted to the sum of two Gaussian distributions (purple line). The individual components are shown as the yellow and orange lines.

tionality constant that depends on the cross-sectional area and the density of states at the Fermi level of the electrodes, $m$ corresponds to the electron mass, and $\phi$ is the work function of the material. Since $G_{a}$ is the conductance in the tunneling regime immediately before the jump to contact, its logarithm $\log \left(G_{a}\right)$ [here $\log$ denotes the common logarithm (base 10)] is proportional to the distance between the electrodes from which the jump occurs. When the $G_{a}$ axis is plotted on a logarithmic scale, the density plot corresponding to the formation of $\mathrm{Au}$ contacts reveals shapes of the maxima that can be more easily interpreted than those previously reported in Ref. [7].

\section{B. Data analysis}

The projections of the density plot data on both $\log \left(G_{a}\right)$ and $G_{b}$ axes (Fig. 2) can be fitted to a sum of Gaussian peaks. This suggests that the density plot is formed by a number of maxima which are normally distributed in both variables. Therefore, we fit the data to the sum of three bivariate normal distributions, sketched as ellipses in Fig. 2, and labeled $D 1, D 2$, and $D 3$, with different relative probabilities $p$. Each of these distributions is described by the expression

$$
f(x, \mu, \Sigma)=\frac{1}{\sqrt{|\Sigma|}(2 \pi)^{2}} e^{-\frac{1}{2}(x-\mu)^{t} \Sigma^{-1}(x-\mu)},
$$

where $\quad x=\left(\log \left(G_{a}\right), G_{b}\right), \quad \mu=\left(\mu_{a}, \mu_{b}\right), \quad$ and $\quad \Sigma=$ $\left(\begin{array}{cc}\sigma_{a}^{2} & \rho \sigma_{a} \sigma_{b} \\ \rho \sigma_{a} \sigma_{b} & \sigma_{b}^{2}\end{array}\right) . \mu_{i}$ and $\sigma_{i}$ represent the two-dimensional (2D) equivalents of the unidimensional mean and standard 
TABLE I. Parameters obtained by fitting density plots of $\mathrm{Au}, \mathrm{Ag}$, and $\mathrm{Cu}$ to the sum of three bivariate normal distributions, namely $D 1$, $D 2$, and D3. $p$ represents the relative probability of each distribution. For simplicity, we denote $\mu_{\log \left(G_{a} / G_{0}\right)}$ and $\sigma_{\log \left(G_{a} / G_{0}\right)}$ as $\mu_{a}$ and $\sigma_{a} . \mu_{a}$ and $\mu_{b}$ thus represent the mean values of distributions in the $\log G_{a}$ and $G_{b}$ axes, respectively. $\sigma_{a}$ and $\sigma_{b}$ are the corresponding standard deviation, and $\rho$ represents the correlation between the two axes. Conductance values are given in quantum units of conductance, $G_{0}=$ $2 e^{2} / h$.

\begin{tabular}{|c|c|c|c|c|c|c|}
\hline & $p(\%)$ & $\mu_{a}$ & $\begin{array}{l}\mathbf{A u} \\
\mu_{b}\end{array}$ & $\sigma_{a}$ & $\sigma_{b}$ & $\rho$ \\
\hline D1 & 58 & -1.2 & 0.9 & 0.4 & 0.2 & 0.3 \\
\hline D2 & 32 & -1.2 & 1.0 & 0.4 & 0.05 & 0.3 \\
\hline \multirow[t]{3}{*}{ D3 } & 10 & -1.1 & 1.7 & 0.4 & 0.3 & 0.2 \\
\hline & & & Ag & & & \\
\hline & $p(\%)$ & $\mu_{a}$ & $\mu_{b}$ & $\sigma_{a}$ & $\sigma_{b}$ & $\rho$ \\
\hline D1 & 52 & -0.6 & 1.1 & 0.2 & 0.2 & 0.4 \\
\hline D2 & 30 & -0.9 & 1.0 & 0.2 & 0.08 & 0.5 \\
\hline \multirow[t]{3}{*}{ D3 } & 18 & -0.6 & 1.9 & 0.2 & 0.2 & 0.4 \\
\hline & & & $\mathrm{Cu}$ & & & \\
\hline & $p(\%)$ & $\mu_{a}$ & $\mu_{b}$ & $\sigma_{a}$ & $\sigma_{b}$ & $\rho$ \\
\hline D1 & 57 & -0.6 & 1.0 & 0.3 & 0.2 & 0.12 \\
\hline D2 & 29 & -0.8 & 1.0 & 0.3 & 0.08 & 0.2 \\
\hline D3 & 14 & -0.6 & 1.8 & 0.3 & 0.2 & 0.2 \\
\hline
\end{tabular}

deviation, respectively, and $\rho$ is the correlation parameter between variables $\log G_{a}$ and $G_{b}$. A " $t$ " superscript denotes vector transposition.

\section{EXPERIMENTAL RESULTS}

The experiments and analysis described in the previous section were repeated during the fabrication of over 2000 contacts made of $\mathrm{Au}, \mathrm{Ag}$, or $\mathrm{Cu}$. The output fitting parameters for the three materials are summarized in Table I. The characteristic parameters of the distributions can be represented graphically by an ellipse (for example, as the overlays in Fig. 2). The center of the ellipse $\left(\mu_{a}, \mu_{b}\right)$ represents the $\left(\log G_{a}, G_{b}\right)$ position of the mean of the distribution, and the axes of the ellipse represent the standard deviations $\left(\sigma_{a}, \sigma_{b}\right)$ in the respective conductance axis. The tilt of the ellipse is proportional to the correlation $(\rho)$ between the two variables. The identification of three maxima is in good agreement with Ref. [7] for Au. The improved analysis thus provides an opportunity to revise the previous results and carry out a more precise quantitative assessment of the data.

In analogy with Ref. [7], we find an isolated distribution with a low probability of occurrence, well above $G_{0}$ (labeled $D 3$ in Fig. 2), while at around $1 G_{0}$ we find the sum of two distributions. Here we disentangle those two distributions and provide an estimate of their relative probabilities $(p)$. The envelope of distribution $D 1$ encloses more than 50\% of the data, while $D 2$ contains around $30 \%$. In this instance, the results for all three materials are similar.

Moreover, on comparing the three materials, we discover a striking result: there is an important difference between the jump distance of $\mathrm{Au}$ versus $\mathrm{Ag}$ and $\mathrm{Cu}$, represented by their mean values of $\log \left(G_{a} / G_{0}\right)$ denoted for simplicity as $\mu_{a}$. This is the focus of a separate study [21], in which we show that the origin of this phenomenon can be traced to the different strengths of relativistic effects in these materials.

Besides the information given by the mean of each distribution, the standard deviation also provides a measure of the dispersion in each. Even if the dispersion in the $\log \left(G_{a}\right)$ values $\left(\sigma_{a}\right)$ is much larger in the case of $\mathrm{Au}$, the ratio between dispersion and the mean value of jump distance $\sigma_{a} / \mu_{a}$ is similar for a given dispersion, for all three materials $(0.33$ for $D 1$ in $\mathrm{Au}$ and $\mathrm{Ag}$, for example). This implies that the variations in the distance of jump seem to occur within a fixed percentage of the central mean value. Our interpretation of this is that the mean value of jump distances is strongly affected by the changes in the interelectrode binding potential that we attribute to the relativistic corrections [21], but the large dispersion in distances of jump originates instead from the very different geometries that the electrodes can adopt. Following the work of Trouwborst et al. [8], different geometries present different intraelectrode elastic constants and thus different distances of jump. If these variations in geometry/elasticity are similar for the three materials, they will result in a higher dispersion for the system, with a larger mean value, but the ratio dispersion/mean should remain similar.

While the dispersion in conductances before jump $\left(\sigma_{a}\right)$ remains similar in all three distributions for each material, remarkably, the dispersion in $G_{b}\left(\sigma_{b}\right)$ exhibits significant differences. Distribution $D 1$, in contact conductance $G_{b}$, is rather broad, while distribution $D 2$, the second most probable, exhibits a rather narrower dispersion in this parameter, as is evident from the widths of the ellipses in the $G_{b}$ axis $\sigma_{b}$. This point will be discussed further in light of atomistic simulations, but it already suggests that the conductance in contact of one of the distributions is considerably less sensitive to geometrical variations than the other.

Finally, we note that the correlation between $G_{a}$ and $G_{b}$, $\rho$ (visible from the tilt of the ellipses), is very similar not only for the three distributions, but also for all three materials, indicating a slight tendency for contacts associated with shorter jump distances to exhibit higher conductances.

Besides the notable discrepancies in $\mu_{a}$, a comparison of the metals also yields a number of subtle differences that are connected to the longer jump distance in the case of $\mathrm{Au}$. First, the means $\mu_{a}$ of $D 1$ and $D 2$ for Au occur at about the same distance, while that of $D 3$ has a slightly different value. However, for $\mathrm{Ag}$ and $\mathrm{Cu}$, distributions $D 1$ and $D 3$ are centered at similar values of $\log G_{a}$, while the contacts corresponding to $D 2$ are established from a greater jump distance. Regarding the value of $\mu_{\mathrm{b}}$, note the lower conductance value for $D 1$ in the case of Au with respect to the other two distributions, as well as with respect to the corresponding values for $\mathrm{Ag}$ or $\mathrm{Cu}$. Although differences between $D 1$ and $D 2$ are small and perhaps within error margins, this behavior is expected for the more "stretched out" structures formed in Au [21].

\section{MOLECULAR-DYNAMICS SIMULATIONS AND $A b$ Initio CALCULATIONS}

\section{A. Methodology}

We have not found any analysis of experimental measurements of electronic transport in the literature that provide 


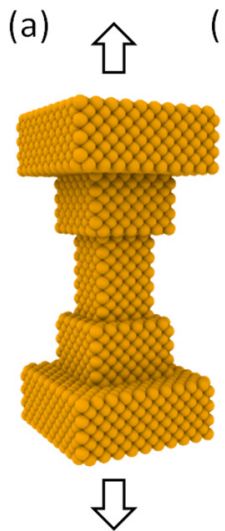

Seed (b)

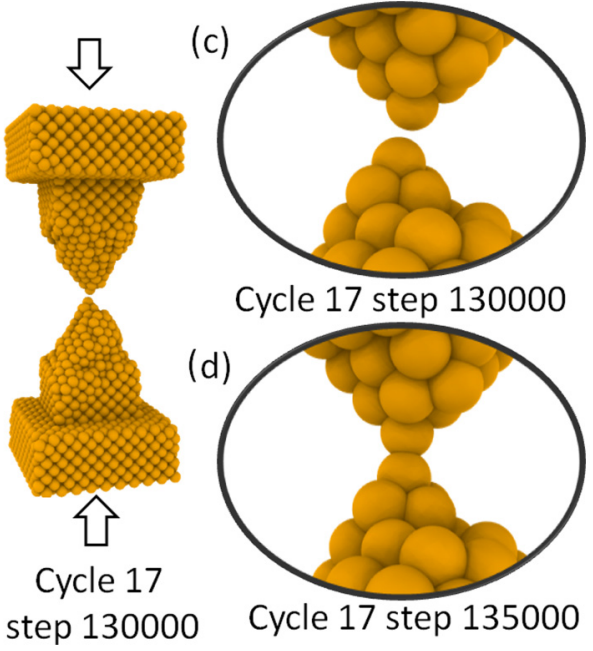

FIG. 3. Snapshots of a gold nanocontact at different times during a molecular-dynamics simulation. (a) Initial structure; arrows in (a) and (b) indicate the direction of elongation or compression. Panel (c) shows a zoom-in of panel (b), which is the step immediately before the contact shown in panel (d) has formed.

information about the geometry at or the instant just before contact is established. Therefore, in order to have an appreciation of the importance of the configuration of the atoms in the immediate vicinity of few-atom contacts, we simulate the experiments by means of classical molecular dynamics (CMD) and first-principles quantum transport calculations. An alternative approach is used in Refs. [22,23], in which a potential energy surface is calculated as an adiabatic trajectory by DFT. There, metal junctions composed of small opposing fragments of $\mathrm{Au}, \mathrm{Ag}$, or $\mathrm{Cu}$ are elongated/separated in small steps, with a geometry optimization at each step.

Molecular-dynamics simulations are, however, based on solving Newton's second law for all the atoms, as they evolve from their initial positions. In such simulations, the potential used to model interactions between the atoms is semiempirical [24]. The initial structure in the present work is independent of the metal and consists of 4736 atoms, oriented along the [100] crystallographic direction, as shown in panel (a) of Fig. 3. We do not consider stacking along [111] in the loading direction in our simulations. The most energetically stable exposed surface layers of $\mathrm{Au}, \mathrm{Ag}$, and $\mathrm{Cu}$ are stacked along [111], because atoms in the exposed surface layers have more nearest neighbors than in any other crystallographic direction. Furthermore, during training of our nanocontacts, even though the temperature is low, the cyclic loading process provides enough energy for the atoms on the electrode tips to attain their most energetically stable configurations [25]. Therefore, for $\mathrm{Cu}, \mathrm{Ag}$, and $\mathrm{Au}$ nanocontacts, this means having [111] layers on the exposed faces of the pyramid-shaped tips. Since these faces are slanted relative to the direction of motion of the tips, the crystallographic direction in the loading direction is more likely along [001].

The result of solving Newton's second law for the system in Fig. 3 is that we can obtain the classical trajectories of all the atoms in the structure as it is ruptured and brought back into contact over many cycles. Extracting from these trajectories, then, the structure at first contact, as well as the one immediately before it, will, via DFT transport calculations [18-20], yield the conductance at the moment that contact is reestablished.

As mentioned above, all the simulations involving $\mathrm{Au}$, $\mathrm{Ag}$, or $\mathrm{Cu}$ are based on the same initial seed structure. The simulations are run in a way that reproduces cyclic loading of the nanowire in analogy with a typical STM or mechanically controllable break junction (MCBJ) experiment. This is also an approach that was followed in our previous works $[6,26]$. The interactions between the metal atoms are modeled by the semiempirical, embedded-atom method (EAM) potential [27]. All the simulations have been realized by means of the large-scale atomic/molecular massively parallel simulator (LAMMPS) [28,29]. The potential parameters used for $\mathrm{Au}, \mathrm{Ag}$, and $\mathrm{Cu}$ in this work are taken from Ref. [30]. The potential itself is derived in Ref. [31].

Additionally, in order to mimic the conditions of the experiment as closely as possible, we simulate at the boiling point of liquid helium, $4.2 \mathrm{~K}$. The Nose-Hoover thermostat $[32,33]$ serves to maintain the temperature constant during the cycles of retraction and approach of the nanoelectrodes in the simulations. Thermostatting is performed every 1000 simulation time steps, the time interval that is recommended by the developers of LAMMPS [29].

The atoms that are located in the first three crystallographic planes from the top of the initial seed structure, as well as the corresponding three planes at the bottom, are pinned to their equilibrium bulk lattice positions so as to constrain their relative positions. The remaining atoms respond dynamically to the bulk motion of these "frozen" planes. Afterward, the entire structure is stretched lengthwise (vertically) by moving the frozen layers in opposite directions at a constant speed of $\sim 1 \mathrm{~m} / \mathrm{s}$. The arrows in Fig. 3, panels (a) and (b), illustrate the directions of the applied forces on both ends (top/bottom) during contact rupture and formation. A speed of $\sim 1 \mathrm{~m} / \mathrm{s}$ may be many orders of magnitude greater than that employed in the experiments, but we argue that there is enough time for the structures to reach equilibrium, and not merely metastable states, because this speed is at least three orders of magnitude lower than the speed of sound in the bulk metals [16]. The low temperature used in our simulations also ensures that processes that would otherwise be important at microsecond time scales, such as surface diffusion, remain negligible. In fact, at $4.2 \mathrm{~K}$, surface diffusion is inhibited by activation energies that are three to four orders of magnitude higher than the thermal energy of the atoms [34].

To perform cyclic loading in CMD, the simulation domain is divided longitudinally into slices of equal height, corresponding to the interlayer spacing within the bulk crystal. In a facecentered-cubic crystal, this spacing is half the lattice parameter along the [100] crystallographic axis. The slice containing the fewest atoms then corresponds to the minimum cross section of the nanocontact. Hence, the structure is stretched until the minimum-atom slice and either of the slices adjacent to it no longer contain any atoms, as shown in Fig. 3(c). At this point, the motion is reversed and the two ruptured tips are brought back together at the same speed with which the structure was first broken. When the minimum-atom layer contains more than 15 atoms, the motion is reversed once more 
and the nanocontact is stretched until it breaks. This process is repeated at least 20 times. To clarify our terminology, we denote by one "cycle" a single rupturing and re-forming of the contact. It is crucial in our simulations to know at which time step during the approach first contact occurs. We detect this moment by monitoring the value of the minimum cross section, which happens when there are more than zero atoms in the contact cross section. This means that contact has been (re)established. Incidentally, the semiempirical potentials describing the interactions between the atoms in the simulations lead to first-contact distances ranging up to half-way between first and second neighbors in a bulk fcc lattice: $\sim 3.5 \AA$ in the cases of $\mathrm{Au}$ and $\mathrm{Ag}$, and $\sim 3.0 \AA$ in the case of $\mathrm{Cu}$. In past works, this has also been used as the criterion to identify the moment of first contact $[9,25]$. Figures 3(c) and 3(d) show the structure prior to and immediately after first contact, respectively.

Finally, to calculate the conductance of structures extracted from molecular-dynamics simulation trajectories, we have used the electronic transport code ANT.G [35], which depends on DFT parameters calculated by GAUSSIAN09 [36]. The structures obtained from CMD contain more than 4000 atoms. Therefore, in order to compute the conductance of these structures within a reasonable time via DFT calculations, it has been necessary to trim the region of interest down to around 500 atoms, keeping only those atoms that lie within a box smaller than the original simulation domain, and centered on the region of first contact, or minimum cross section. However, obtaining accurate conductance values required, in addition, that we assign a larger basis set of 11 valence electrons to $\sim 40$ atoms in the contact region. The rest of the atoms were assigned a basis set of one valence electron.

\section{B. Molecular-dynamics results}

For the analysis of the CMD results obtained after 20 cycles of contact rupture and formation, we have used a simple algorithm that counts the number of atoms in layers spaced vertically along the simulation domain. By keeping in mind that the three layers on opposite ends of the structures remain "frozen" internally during the simulations, i.e., that the lattice parameter of these layers stays fixed at the bulk value, we discretize the entire structure into a number of layers half the bulk lattice parameter in thickness. As lattice parameters, we used $4.08 \AA$ for $\mathrm{Au}$ and $\mathrm{Ag}$, and $3.61 \AA$ for Cu. Consequently, during an approach (contact formation) phase, for example, we count, at every step, the number of atoms in each layer. Figure 4(a) shows how the layers are distributed along the length of the nanocontact. The plot in Fig. 4(c) was constructed by counting the number of atoms in each layer. Thus, in principle, a zoom-in of the atoms in the minimum cross section in Fig. 4(a), located somewhere between layers 24 and 29, should lead us to conclude that the contact type is "4-1-1-4." Panel (b) is such a zoom-in of panel (a) and shows clearly what the contact type is. It therefore confirms, via visual inspection, the result inferred from panel (c). The trace in Fig. 4(d) has been constructed by plotting the minimum of the parabola in (c) against the simulation time step. The resemblance to an experimental conductance trace is, at the very least, suggestive. Furthermore, we would like to point out that panel (c) contains more information than is used for the purposes of the present
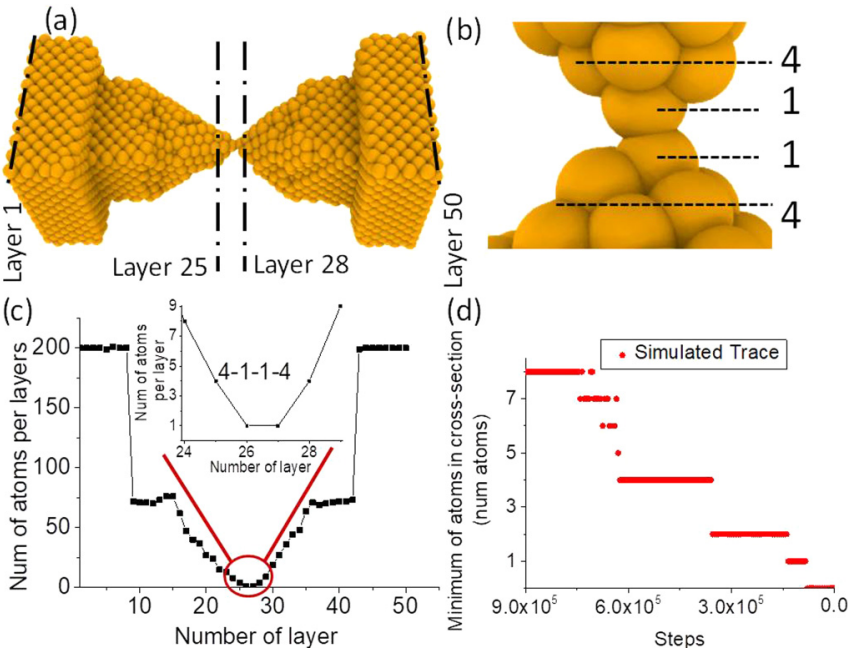

FIG. 4. (a) The atomic-sized gold contact simulated via MD, with the layer positions indicated by dashed-dotted lines. (b) A zoom-in of (a), showing the type of contact identified by our algorithm, the results of which are shown in (c). (d) The number of atoms in the minimum cross section as a function of simulation time step. The inset in (c) is a zoom-in that shows when, during the simulation, exactly one atom remains in the minimum cross section.

article. In fact, such a plot can also give us an idea about the evolution of the sharpness of the contact. For example, blunt electrodes should lead to a steeper slope in the parabola than sharper tips. This tool could pave the way to an improved analysis of the evolution of the contact in CMD, one that renders direct visualization unnecessary. In addition, a better counting algorithm could take advantage of it. All the results in Fig. 4 have been extracted from cycle 5 of the simulation involving $\mathrm{Au}$, in which contact occurs at time step 85000 .

The methodology followed to count atoms in the cross section is not unique. Other algorithms, such as the one developed by Bratkovsky et al. [14], do not count an integer number of atoms and neighbors in the contact minimum cross section. In this work, we have modified the Bratkovsky algorithm to suit our purposes and count an integer number of atoms in the layers. We are well aware of the limitations of our method; therefore, to obtain complementary information, we calculate the conductance of the CMD structures via DFT, and if, in the worst of cases, it differs very much from the expected value, we recheck the structure by visual inspection, and where necessary reassign an appropriate contact type.

Thus, we have employed the approach summarized in Fig. 4 to study the three metals and the 20 cycles of contact rupture and formation they undergo during the simulations. By following the procedure outlined in the next paragraph, we have been able to identify different types of contacts as well as their first neighbors, as detailed in Fig. 5.

Our criterion for identifying the contacts as single, double, or triple involves counting the number of atoms in the minimum cross section between the leads, at the very moment when the corresponding layers become populated during the simulation. All three contact types can occur in a monomeric or dimeric configuration, as illustrated in Fig. 5. The "low" and "high" coordination designations, irrespective of whether the contacts 


\section{Single Monomer Low}

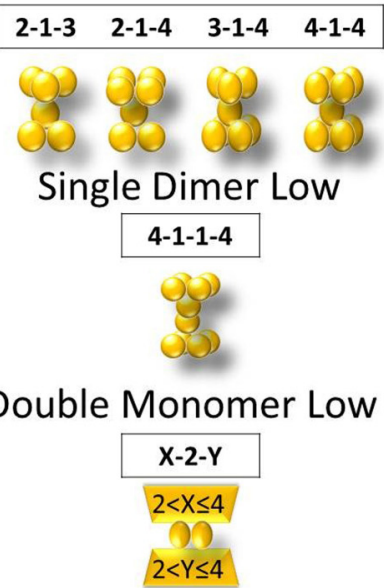

Double Dimer Low

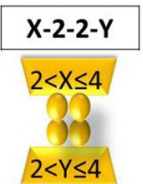

Triple Monomer Low

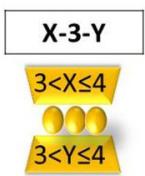

FIG. 5. Illustration of the different types of contacts. Left column: low-coordination first-neighbor contacts. Right column: highcoordination first-neighbor contacts. Each of the single, double, or triple contacts can also occur as monomers or dimers.

are monomeric or dimeric, depend on the number of first neighbors found by our algorithm, on either side of the minimum-atom layer. We have established the limit of first neighbors based on an exposed [001] fcc surface layer, which, as is known, is puckered by fourfold hollows, such that an adsorbed atom will have four first neighbors immediately beneath it [9]. Therefore, "low" coordination means equal to or less than four first neighbors in both electrodes. As soon as the limit of four first neighbors is exceeded in one of the electrodes, that side is designated as "high" coordination. Figure 5 summarizes the typical contacts encountered in our simulations.

For some of the contacts that form, there is an indeterminate number of possible configurations, and therefore to simplify the statistical analysis, we use an $\mathrm{X}$ to represent combinations with more than four first-neighbor atoms. Likewise, we use a $\mathrm{Y}$ in combinations where the number of first-neighbor atoms is in a similar range to, or is larger than, $\mathrm{X}$ (see Fig. 5).

Hence, we have simulated contact evolution over continuous loading cycles, and we studied the electronic transport during contact formation by means of DFT calculations. After 20 cycles, some of the contact types are reproduced several times while other contact types appear only once. Table II records, for every cycle, the contact type and number of
TABLE II. Conductance values obtained for CMD snapshots selected by our first-neighbor visual correction. The colors blue, red, and green represent $\mathrm{Au}, \mathrm{Ag}$, and $\mathrm{Cu}$, in that order. $\mathrm{X}$ and $\mathrm{Y}$ are any value bigger than 4 .

\begin{tabular}{lcccccc}
\hline \hline Cycle & Type & $\mathbf{G}[\boldsymbol{G}(\mathbf{0})]$ & Type & $\mathbf{G}[\boldsymbol{G}(\mathbf{0})]$ & Type & $\mathrm{G}[G(\mathbf{0})]$ \\
\hline 1 & $4-1-5$ & 1.26 & $6-2-6$ & 2.43 & $6-3-6$ & 3.25 \\
2 & $3-1-3$ & 0.72 & $\mathrm{X}-2-\mathrm{Y}^{* *}$ & 0.69 & $6-3-4$ & 1.71 \\
3 & $\mathrm{X}-3-\mathrm{Y}$ & 2.45 & $4-1-2$ & 0.78 & $4-1-3$ & 0.84 \\
4 & $2-1-4$ & 1.58 & $3-1-2$ & 0.95 & $8-3-6$ & 2.31 \\
5 & $4-1-1-5$ & 0.73 & $6-2-4$ & 1.55 & $5-4-8$ & 2.54 \\
6 & $\mathrm{X}-3-\mathrm{Y}$ & 2.76 & $8-2-5$ & 1.72 & $8-3-4$ & 1.58 \\
7 & $4-3-\mathrm{Y}$ & 2.80 & $8-2-4$ & 1.72 & $5-1-2$ & 1.04 \\
8 & $4-1-2$ & 0.84 & $5-1-5$ & 1.14 & $6-3-4$ & 2.59 \\
9 & $7-3-4$ & 2.34 & $6-2-6$ & 1.80 & $5-1-4$ & 0.58 \\
10 & $3-1-4$ & 1.45 & $6-2-2-6$ & 1.47 & $4-1-1-6$ & 0.83 \\
11 & $\mathrm{X}-2-\mathrm{Y}$ & 2.02 & $6-2-4$ & 1.85 & $6-2-4$ & 1.29 \\
12 & $3-1-2$ & 1.25 & $6-2-4$ & 1.72 & $5-4-6$ & 3.14 \\
13 & $\mathrm{X}-2-\mathrm{Y}$ & 2.22 & $6-2-6$ & 1.71 & $4-3-5$ & 2.47 \\
14 & $5-1-1-3$ & 0.86 & $5-1-1-5$ & 0.99 & $* * *$ & 1.32 \\
15 & $4-2-3$ & 1.15 & $5-1-1-5$ & 0.75 & $4-2-4$ & 2.02 \\
16 & $4-1-1-5$ & 1.27 & $5-1-1-5$ & 0.61 & $4-1-2$ & 1.00 \\
17 & $4-1-1-4$ & 0.88 & $6-2-6$ & 1.66 & $4-3-7$ & 2.34 \\
18 & $2-1-4$ & 1.63 & $6-2-6$ & 1.72 & $4-1-2$ & 1.14 \\
19 & $3-1-3$ & 1.35 & $6-2-6$ & 1.82 & $8-3-5$ & 1.47 \\
20 & $4-2-6$ & 2.20 & $6-2-6$ & 1.75 & $\mathrm{X}-3-\mathrm{Y}$ & 1.72 \\
\hline \hline
\end{tabular}

first-neighbor atoms according to the nomenclature outlined in Fig. 5. In the same table, we have corrected the type of contact through visual inspection. Raw data about the type of contact, i.e., in the absence of visual inspection, are collected in Table IV in the Appendix. Finally, the double and triple asterisks in Table II refer to those curious cases in which two or three atoms close to forming a contact contribute to the conductance across the junction, but directly via tunneling.

\section{DFT calculations based on CMD simulations}

All the CMD frames that have been analyzed from the point of view of the geometry in Table IV have also been analyzed via DFT conductance calculations. The results are shown in Table II, and they are also included in Fig. 6.

The structures obtained from CMD simulations, which are limited in their ability to predict realistic structures, require interpretation via electronic transport calculations (if meaningful comparisons with the experimental results are to be made). Following this, upon comparing the calculated conductance and experimental density plots, we can extract information about the type of contact that is formed as well as the configuration of the first-neighbor atoms surrounding it. The electronic transport across all the structures has been calculated by means of ANT.G [35], which interfaces with GAUSSIAN09 [36]. We have grouped the various contacts by type, and their mean conductance values and standard deviations are plotted in Fig. 6 as dots and vertical bars, respectively.

\section{DISCUSSION}

In this work, our aim is to find the origin of the subtle differences between materials, and to identify the properties of 


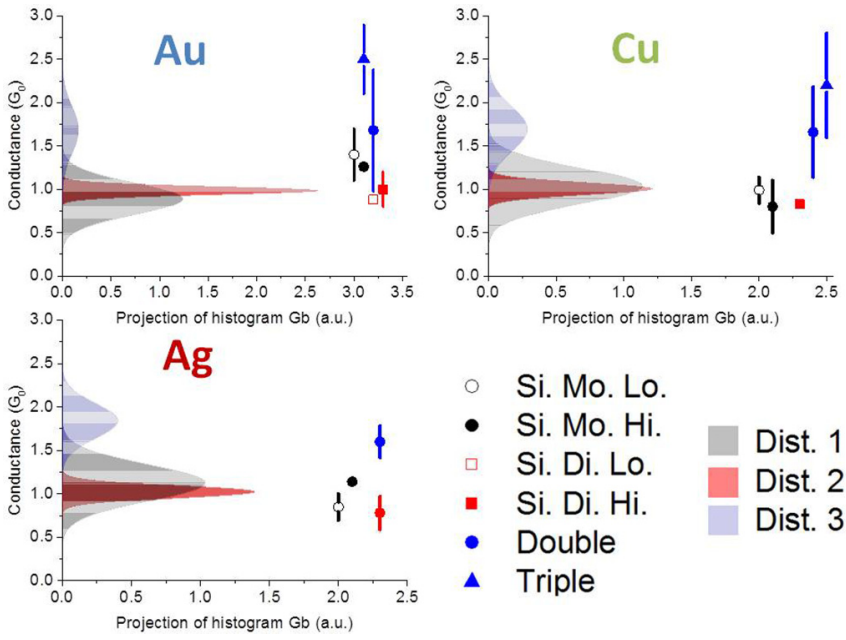

FIG. 6. Projection of experimental $G_{b}$ values vs number of counts, for $\mathrm{Au}, \mathrm{Ag}$, and $\mathrm{Cu}$. Data points and error bars: the conductance and standard deviation of the various simulated contacts. "Single" is denoted by Si, followed by Mo or Di, for either "monomer" or "dimer." Hi and Lo represent high and low coordination.

types of contacts defined by their specific geometry. Elsewhere, we prove that relativistic effects are responsible for the large discrepancy between the jump-to-contact distances of $\mathrm{Au}$ and $\mathrm{Ag}$ [21], represented by the respective means of their $G_{a}$ values.

To approach this problem, we use CMD as a tool to visualize the moment of first contact and identify the number and arrangement of the first neighbors. We cannot rely on CMD in the case of tunneling because the potentials only account indirectly for the effects of electrons, and hence relativity, and then only very crudely. Furthermore, it is not possible, experimentally, to know the structure and geometry of the electrodes in the tunneling regime. In CMD, the structure before contact is, at times, preserved in contact, as illustrated in Figs. 3(c) and 3(d). At other times, significant rearrangements occur and the before-contact structures are no longer preserved. For this reason, we confine our analysis to the first neighbors in the contact regime.

The conductance values obtained via DFT from the CMD structures are summarized in Table III. The comparison of these results with the experimental distribution of values (Fig. 6) allows us to interpret our results in terms of the simulated geometry of the contacts. Double and triple contacts are simplified in Fig. 6, i.e., we do not distinguish between high or low, or monomer or dimer. Thus, the blue dot and triangle represent mean values, and their error bars represent the standard deviations obtained through grouping.

In spite of the reduced statistics (we have performed 20 loading cycles in CMD, on each metal), the distribution of conductance values from the simulated geometries, classified as monomer, dimer, or higher-order contacts, largely coincides with the three distributions fitted to the experimental data. These results show that distribution $D 3$ likely arises from a combination of double- and triple-contact structures (blue triangles and circles in Fig. 6). Because of the configuration
TABLE III. The first column refers to the type of contact that has been formed: single, double, or triple. In the second column, each of these contact types is further classified into monomer or dimer. The " $L$ " and " $H$ " designations under the column heading "Coord" refer to Low and High coordinations, respectively. Then, the following three columns show the average conductance values and their standard deviations for gold, silver, and copper, respectively. Configurations not found in molecular-dynamics simulations are left blank.

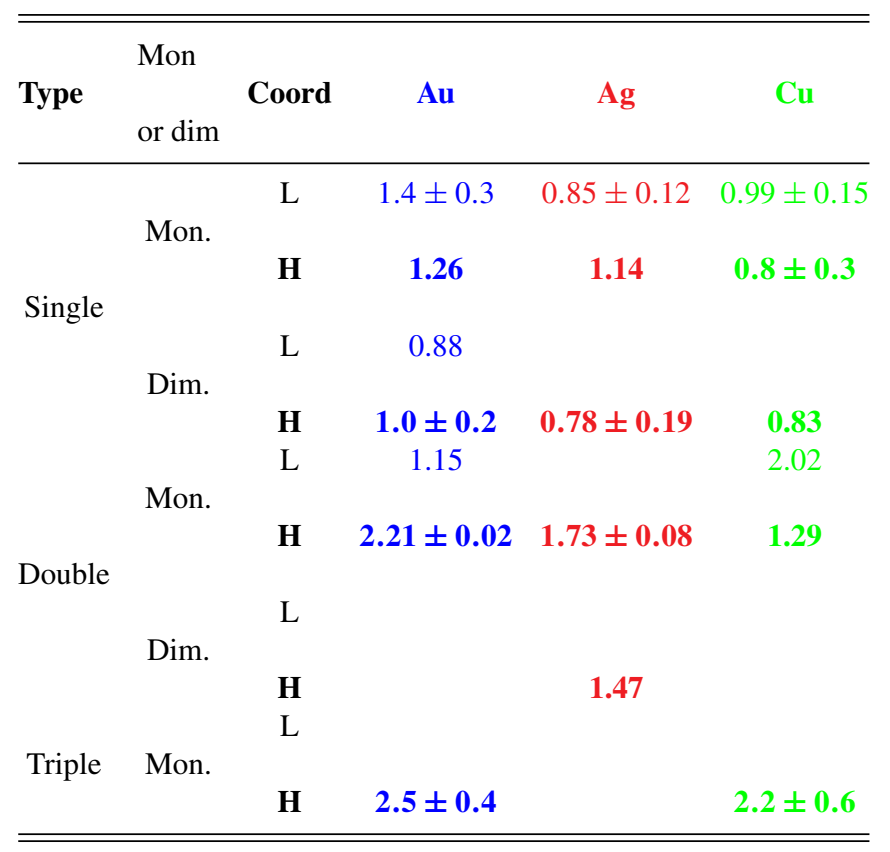

of these contacts, there is a wide distribution in conductance values, as can be seen also from the experimental data. Among these structures, we have identified, through conductance calculations, the triple contact, whose conductance values are in the $2-3 G_{0}$ range.

Here we propose that the $D 1$ and $D 2$ distributions can be assigned to monomers and dimers, respectively. Experimentally, there is a large difference in dispersion of the two distributions, with $D 1$ showing a much larger dispersion than $D 2$ in all three metals. Our calculations also yield a higher dispersion in conductance for monomers than for dimers, if all types of monomers are considered (Table II, Fig. 6), and therefore we could associate these contacts with distributions $D 1$ and $D 2$, respectively, in agreement with the assignment by Untiedt et al. [7]. This argument is further supported in terms of the geometry of these contacts; the values listed in Table II exemplify how variations in the number of neighbors for a dimer have little repercussion on the value of the conductance, therefore a narrow profile is to be expected. For a monomer, on the other hand, the number of neighbors results in large changes in conductance and a wider distribution.

Furthermore, the simulations also allow us to classify the contacts into high and low coordination. This classification does not provide much additional interpretation of the experimental results due to the reduced statistics, but it does highlight the determining role of coordination on the conductance of atomic contacts. 


\section{SUMMARY}

We have introduced a statistical approach that permits identifying properties of atomic-sized contacts with greater precision. In this way, it has been possible to study, in detail, the process of formation of $\mathrm{Au}, \mathrm{Ag}$, and $\mathrm{Cu}$ nanocontacts. Moreover, such an analysis has allowed us to identify with higher precision the distribution of values of conductance associated with different geometries, and also to extract information on the distance of contact formation for those geometries. Furthermore, we have used molecular dynamics to simulate the formation of atomic-sized contacts in STM/MCBJ experiments. These simulated contacts were, in turn, analyzed by means of an improved methodology that permits classifying their type and finding the number of first-neighbor atoms in their immediate vicinity. DFT transport calculations on the simulated structures provided a means of comparing theoretical results with the experimental data. We have demonstrated that the type of contact and the geometry of its first neighbors (shape, distance between first-neighbor atoms, and between them and the atomic contact itself) play decisive roles in electronic transport across the simulated contacts. Through a combination of the above three methods, we have found that the electronic transport across the atomic-sized contacts depends crucially on the number of first-neighbor atoms.

\section{ACKNOWLEDGMENTS}

This work has been funded from the Spanish Ministerio de Educación y Ciencia through Grants No. FIS2013-47328 and No. MAT2016-78625 and the Conselleria d'Educaci, Investigaci, Cultura i Esport de la Generalitat Valenciana, PROMETEO/2017/139. C.S. gratefully acknowledges financial support from SEPE Servicio Público de Empleo Estatal. W.D. acknowledges funding from the National Research Foundation of South Africa through the Innovation Doctoral scholarship programme, Grant UID 102574. W.D. also thanks J. Fernandez-Rossier and J.J. Palacios for fruitful discussions.
TABLE IV. Results produced by modified Bratkovsky algorithm to count the integer number of atoms. The colors refer to different materials: $\mathrm{Au}$ (blue), $\mathrm{Ag}$ (red), and $\mathrm{Cu}$ (green).

\begin{tabular}{lcccccc}
\hline \hline Cycle & kStep & Type & kStep & Type & kStep & Type \\
\hline 1 & 3090 & $10-1-2$ & 1545 & $5-2-5$ & 715 & $6-3-8$ \\
2 & 145 & $4-1-2$ & $1410^{*}$ & $\mathrm{X}-2-\mathrm{Y}^{*}$ & 540 & $6-3-4$ \\
3 & 285 & $3-2-5$ & 405 & $6-1-2$ & $220^{*}$ & $4-1-3 *$ \\
4 & 605 & $2-1-4$ & 395 & $3-1-2$ & 1055 & $2-1-6$ \\
5 & 85 & $4-1-1-4$ & 250 & $6-2-6$ & 335 & $5-2-3$ \\
6 & 58 & $4-3-11$ & 210 & $5-3-5$ & 185 & $4-1-3$ \\
7 & $275 *$ & $4-3-Y^{*}$ & 320 & $6-3-5$ & 610 & $6-2-2-7$ \\
8 & 160 & $6-1-2$ & 210 & $6-2-6$ & 285 & $3-1-4$ \\
9 & 425 & $7-3-4$ & 255 & $6-3-5$ & $165 *$ & $5-1-4 *$ \\
10 & 240 & $2-1-4$ & 315 & $6-3-5$ & 525 & $4-1-4$ \\
11 & 400 & $4-2-5$ & $255 *$ & $6-2-4 *$ & 385 & $3-2-4$ \\
12 & $435 *$ & $3-1-2 *$ & 320 & $5-3-5$ & 1060 & $3-1-6$ \\
13 & 375 & $7-2-3$ & 255 & $6-3-5$ & $720 *$ & $4-3-5 *$ \\
14 & 365 & $7-1-1-2$ & 240 & $6-2-6$ & $1260 *$ & $\mathrm{X}-4-\mathrm{X}^{*}$ \\
15 & 200 & $5-2-3$ & 280 & $4-1-6$ & 180 & $8-2-2-4$ \\
16 & 185 & $2-1-3$ & 305 & $6-2-6$ & 225 & $5-1-1-3$ \\
17 & 135 & $4-1-1-4$ & 210 & $6-3-5$ & $225 *$ & $4-3-7 *$ \\
18 & 535 & $3-1-2$ & 210 & $6-3-5$ & 265 & $3-1-1-6$ \\
19 & 340 & $3-1-2$ & 355 & $6-3-5$ & 410 & $8-3-5$ \\
20 & 700 & $4-2-6$ & 215 & $6-3-5$ & 160 & $8-3-4$ \\
\hline \hline
\end{tabular}

\section{APPENDIX}

The methodology described in Sec. IV B and illustrated in Fig. 4 has been applied to the three materials during $20 \mathrm{MD}$ rupture-formation cycles. Table IV summarizes the obtained results. It records, for $\mathrm{Au}, \mathrm{Ag}$, and $\mathrm{Cu}$ (in blue, red, and green, respectively), the time step (in kilosteps, or, more precisely, picoseconds) when contact is established as well as the type of first contact that is formed during every cycle. Data marked with asterisks indicate that the algorithm has detected a contact when it has not really occurred. Through visual inspection, we have selected the correct CMD time frame in which contact actually occurred and we also identified the type of contact.
[1] C. Joachim, J. K. Gimzewski, and A. Aviram, Nature (London) 408, 541 (2000).

[2] N. Agraït, A. L. Yeyati, and J. M. Van Ruitenbeek, Phys. Rep. 377, 81 (2003).

[3] J. Wang, H. Guo, J.-L. Mozos, C. C. Wan, G. Taraschi, and Q. Zheng, Phys. Rev. Lett. 80, 4277 (1998).

[4] K. Terabe, T. Hasegawa, T. Nakayama, and M. Aono, Nature (London) 433, 47 (2005).

[5] R. Vardimon, M. Klionsky, and O. Tal, Phys. Rev. B 88, 161404 (2013).

[6] C. Sabater, M. J. Caturla, J. J. Palacios, and C. Untiedt, Nanoscale Res. Lett. 8, 257 (2013).

[7] C. Untiedt, M. J. Caturla, M. R. Calvo, J. J. Palacios, R. C. Segers, and J. M. van Ruitenbeek, Phys. Rev. Lett. 98, 206801 (2007).

[8] M. L. Trouwborst, E. H. Huisman, F. L. Bakker, S. J. van der Molen, and B. J. van Wees, Phys. Rev. Lett. 100, 175502 (2008).
[9] M. A. Fernández, C. Sabater, W. Dednam, J. J. Palacios, M. R. Calvo, C. Untiedt, and M. J. Caturla, Phys. Rev. B 93, 085437 (2016).

[10] J. Kröger, N. Néel, A. Sperl, Y. F. Wang, and R. Berndt, New J. Phys. 11, 125006 (2009).

[11] M. Müller, C. Salgado, N. Néel, J. J. Palacios, and J. Kröger, Phys. Rev. B 93, 235402 (2016).

[12] J. B. Pethica and A. P. Sutton, J. Vac. Sci. Technol. A 6, 2490 (1988).

[13] U. Landman, W. D. Luedtke, N. A. Burnham, and R. J. Colton, Science 248, 454 (1990).

[14] A. M. Bratkovsky, A. P. Sutton, and T. N. Todorov, Phys. Rev. B 52, 5036 (1995).

[15] M. Brandbyge, J. Schiøtz, M. R. Sørensen, P. Stoltze, K. W. Jacobsen, J. K. Nørskov, L. Olesen, E. Laegsgaard, I. Stensgaard, and F. Besenbacher, Phys. Rev. B 52, 8499 (1995). 
[16] M. R. Sørensen, M. Brandbyge, and K. W. Jacobsen, Phys. Rev. B 57, 3283 (1998).

[17] M. Dreher, F. Pauly, J. Heurich, J. C. Cuevas, E. Scheer, and P. Nielaba, Phys. Rev. B 72, 075435 (2005).

[18] J. J. Palacios, A. J. Pérez-Jiménez, E. Louis, and J. A. Vergés, Phys. Rev. B 64, 115411 (2001).

[19] J. J. Palacios, A. J. Pérez-Jiménez, E. Louis, E. San Fabián, and J. A. Vergés, Phys. Rev. B 66, 035322 (2002).

[20] E. Louis, J. A. Vergés, J. J. Palacios, A. J. Pérez-Jiménez, and E. San Fabián, Phys. Rev. B 67, 155321 (2003).

[21] M. R. Calvo, C. Sabater, W. Dednam, E. B. Lombardi, M. J. Caturla, and C. Untiedt, Phys. Rev. Lett. 120, 076802 (2018).

[22] M. S. Hybertsen and L. Venkataraman, Acc. Chem. Res. 49, 452 (2016).

[23] M. S. Hybertsen, J. Chem. Phys. 146, 092323 (2017).

[24] M. P. Allen and D. J. Tildesley, Computer Simulation of Liquids (Oxford University Press, Oxford, 1989).

[25] W. Dednam, C. Sabater, M. A. Fernandez, C. Untiedt, J. J. Palacios, and M. J. Caturla, J. Phys.: Conf. Ser. 574, 012045 (2015).
[26] C. Sabater, C. Untiedt, J. J. Palacios, and M. J. Caturla, Phys. Rev. Lett. 108, 205502 (2012).

[27] M. S. Daw and M. I. Baskes, Phys. Rev. Lett. 50, 1285 (1983).

[28] S. Plimpton, J. Comput. Phys. 117, 1 (1995).

[29] S. Plimpton et al., computer code LAMMPS, publicly available at http://lammps.sandia.gov.

[30] X. Zhou, H. Wadley, R. A. Johnson, D. Larson, N. Tabat, A. Cerezo, A. Petford-Long, G. Smith, P. Clifton, R. Martens et al., Acta Mater. 49, 4005 (2001).

[31] H. Wadley, X. Zhou, R. Johnson, and M. Neurock, Prog. Mater. Sci. 46, 329 (2001).

[32] S. Nosé, Mol. Phys. 52, 255 (1984).

[33] W. G. Hoover, Phys. Rev. A 31, 1695 (1985).

[34] H. Ibach, Physics of Surfaces and Interfaces (Springer-Verlag, Berlin, Heidelberg, 2006), Vol. 12.

[35] J. J. Palacios et al., computer code ANT.G, publicly available at http://www.alacant.ua.es.

[36] M. J. Frisch et al., computer code GAUSSIAN09, Revision C.01, Gaussian, Inc., Wallingford, CT, 2009. 